\title{
RNA Polyadenylation
}

National Cancer Institute

\section{Source}

National Cancer Institute. RNA Polyadenylation. NCI Thesaurus. Code C18947.

The enzymatic addition of a sequence of adenylyl residues at the 3' end of an RNA molecule. 